\title{
LA ATENCIÓN FAMILIAR SEGÚN EL DISEÑO MUSEOGRÁFICO
}

\section{FAMILIAR ATTENTION ACCORDING TO THE MUSEOGRAPHIC DESIGN}

\author{
Keilyn Rodríguez Sánchez ${ }^{1}$ \\ keilyn.rodriguez@ucr.ac.cr
}

Fecha de recepción: 23 de junio de 2015 - Fecha de aceptación: 23 de mayo de 2016

\begin{abstract}
Resumen
El artículo expone un estudio sobre los tipos atención de las familias que visitan la Sala de la Tierra del Museo de los Niños en Costa Rica según los diferentes diseños de objetos museográficos. Mediante un estudio observacional no participante, se concluyó que pese a las diferencias individuales en la atención a los objetos, las familias estudiadas interactúan con objetos cuyo diseño les permita estar juntos, les ofrezca novedad en la secuencia de uso, y les brinde posibilidades multisensoriales de participación. Palabras clave: Museografía educativa. Estudios de visitantes o de audiencia. Pedagogía familiar. Observación no participante.
\end{abstract}

\begin{abstract}
This article refers a study on the attention of the families who visit the La Tierra exhibition in the Children's Museum of Costa Rica, according to the different museographic designs. Through an observational study it was concluded that despite the individual differences, according to the attention on the objects, the families that were studied interact with objects depending on their design and being together. Also, gives them novelty in the sequence of use, and provides them with opportunities to experience a multisensory participation. Keywords: Educational Museography. Visitor Studies/Audience Research. Family Pedagogy. Non Participating Observation.
\end{abstract}

\section{Introducción}

El presente artículo ofrece un análisis de la atención familiar en la visita a la sala museográfica de "La Tierra" del Museo de los Niños de Costa Rica, con el interés de identificar si existen unos objetos en exhibición que captan la atención más que otros según el tiempo que las familias y sus integrantes dediquen a cada uno de ellos. Además, interesa mostrar si se presenta alguna diferencia significativa en la atención focalizada de las familias hacia los objetos museográficos, según las siguientes variables:

- Las personas que integran la familia, y sus particularidades, considerando diferencias generacionales (adulta y joven) de edad (hijos e hijas mayores y menores) y el sexo (mujeres y hombres).

1 Escuela de Antropología, de la Universidad de Costa Rica, Sede Rodrigo Facio. 
- Las características del diseño de cada uno de los dispositivos museográficos (en la sala seleccionada se ubican 13) de la sala de la Tierra en el Museo de los Niños de Costa Rica, lugar donde se realiza la investigación.

- La atención focalizada individual, solitaria y compartida ante los diferentes dispositivos.

De esta manera, se evidencia las relaciones entre los patrones de comportamiento de atención familiar (individual, compartida y conjunta) y los diferentes dispositivos, según sus especificidades en diseño. Por lo tanto, se caracterizan los dispositivos de la sala del Museo con los cuales se asocia la atención focalizada de manera significativa y no como un comportamiento al azar, considerando las demandas cognitivas y sensoriales de cada uno según su diseño. Es importante señalar que la sala de exhibición "La Tierra", es la segunda sala del Museo de los Niños, se encuentra después de la del "Universo y tecnología espacial".

En este artículo, se inicia dando a conocer los conceptos teóricos centrales, luego se ofrece la descripción del método de investigación, y, posteriormente, se expone la sala de la Tierra del Museo de los Niños de Costa Rica, desde su concepción museográfica. Se da a conocer la puesta en escena de dicho espacio mediante una clasificación que considera los objetos o dispositivos que la componen, los objetivos específicos de cada una y se indica las invitaciones cognitivo-sensoriales que según su diseño ofrecen. Se iniciará con una descripción de aspectos generales de la sala para facilitar la comprensión de lo que se desea mostrar.

Es importante destacar que este artículo se desprende de resultados de investigación de la tesis doctoral de la autora (Rodríguez, 2008).

\section{Conceptos teóricos centrales}

Cuando las familias ingresan a la sala de un museo inician un proceso de exploración perceptiva, de tal manera que en un momento determinado, unos objetos logran captar la atención más que otros; a esto se le ha llamado atención individual focalizada, siempre y cuando se sostenga por tres o más segundos, lapso detallado por Colomina (1996).

Posteriormente, es posible que dos o más sujetos focalicen su atención en el mismo dispositivo, esto es lo que se conoce como atención compartida según Mas, (2003). Este comportamiento podría ser la antesala de la atención conjunta (Mas, 2003), la cual consiste en una coordinación de la actividad (verbal o no verbal) entre dos o más sujetos con un objeto (en este caso un dispositivo). En este punto, se puede señalar que la interacción familiar con el objeto ha sido exitosa y constituye la antesala de la co-construcción de sentido sobre el significado cultural que el objeto porta.

Ahora bien, la atención focalizada supone la habilidad de seleccionar o identificar un objeto entre otros, mediante procesos de categorización, mientras que la atención compartida se coordina con otras personas según Mas (2003). También supone que las personas identifican similitudes y diferencias entre los objetos con base en ciertas dimensiones, para localizar las singularidades de los mismos por las características que presentan o no, por lo que su atención se vería atrapada en ellos.

La atención compartida es el acompañamiento de dos o más personas de la familia, ante un objeto de interés común con el cual se está interactuando. Se asume que sucede una coordinación de la atención cuando este comportamiento resulta estadísticamente significativo. La atención individual supone la no existencia para coordinar la atención ante un dispositivo.

\section{Método de investigación}

Se utiliza el método observacional de investigación (Quera, 1997; Bakeman y Gottman, 1997). De esta manera, se realizó una observación directa en la sala durante 4 meses -desde mediados de julio hasta mediados de noviembre del 2005-, la recolección de datos se realizó sólo fines de semana, 
que es cuando la mayoría de las familias visitan el Museo de los Niños en Costa Rica. El tipo familiar seleccionado fue el constituido por madre, padre y dos hijos o hijas de I y II Ciclo escolar, ya que en un estudio previo (Rodríguez, 2008) se determinó que era un 75\% de las familias visitantes. Las familias se identificaron en la entrada al Museo, dónde se les solicitó que firmaran el consentimiento informado para participar del estudio. No se les informó en cuál sala se realizaban las observaciones.

Se elaboró un croquis de la sala que se seleccionó, en el cual se registraron los datos. Con cuatro observadores, se anotó en dicho croquis el tiempo de ingreso (determinado por el primer miembro de familia que ingresa a la sala) el de salida (determinado por el último miembro que sale de la sala), duración en cada dispositivo (tiempo de inicio y tiempo final), el ritual de uso del espacio, el tiempo que cada sujeto duró en cada dispositivo, así como el tiempo que el guía de sala pasó con el sujeto en cada dispositivo, esto para cada uno de los integrantes del grupo familiar. Se realizaron mediciones a 40 familias (160 sujetos, por ser familias de 4 personas cada una) con un error cuadrático medio de 1 segundo y 6 décimas. Una de las limitantes de la investigación es que la muestra debió ser de 60 familias, por falta de recursos y tiempo no se logró, pese a lo anterior, se identificaron diferencias estadísticamente significativas. Los datos se registraron en una base de datos utilizando SPSS, versión 15. La medida del error cuadrático medio se efectuó con la siguiente fórmula: Media (parámetro registro de observador por dispositivo - media registro de observadores por dispositivo) ${ }^{2}$.

Ahora bien, la atención focalizada en términos de medición, y en los términos de este estudio, está definida por el tiempo durante el cual el sujeto se acerca al dispositivo y se dirige hacia él, hasta el momento en que se retira físicamente y orienta su cuerpo hacia otra dirección, se considera atención focalizada a partir de 3 segundos (todas las mediciones se indican en segundos); cuando el sujeto realiza esa acción sin otro miembro de la familia se considera que la atención es focalizada e individual. Cuando los tiempos de los miembros familiares se traslapan en su estadía ante un dispositivo por más de 3 segundos, se considera que la atención es focalizada compartida.

La clasificación de los dispositivos de la sala en estudio, se efectuó con base en las demandas cognitivo sensoriales según el diseño de los mismos y el análisis de los datos obtenidos en las observaciones directas en sala, se hizo mediante t-test, para determinar las diferencias estadísticamente significativas entre las medias.

\section{Hipótesis de trabajo}

Existen diferencias significativas en la atención focalizada de las familias hacia los objetos museográficos, según se indica a continuación:

- Los dispositivos que al utilizarse demandan mayor involucramiento de sentidos, captan más la atención de todos.

- Los dispositivos que son diseñados para leer y observar son más utilizados por adultos e hijos e hijas mayores.

- Los dispositivos que demandan el uso de más sentidos son preferidos por los hijos y las hijas.

- No existe diferencia significativa según sexo y tiempo de atención de los objetos, en las demandas cognitivas y sensoriales.

- Entre los hijos y las hijas mayores y menores, existe diferencia de género en el uso de los dispositivos.

- Los dispositivos que permiten la atención compartida son los preferidos por las familias.

\section{El espacio desde su concepción museográfica: la sala de la Tierra}

Los dispositivos de la sala de la Tierra del Museo de los Niños se describen a continuación según el tipo de invitaciones cognitivo-sensoriales que proponen a los y las visitantes desde su diseño, su nombre, y su objetivo temático: 
Tabla 1

Los dispositivos según sus demandas cognitivo-sensoriales y sus respectivos objetivos

cognitivo-sensoriales
Leer y observar ilustracio-
nes. Cognitivo: utilización de
la memoria y selección de infor-
mación escrita para comprender
la imagen. Compartir con otros
el espacio.
Sensorial: visión.
Comparar un modelo tridi-
mensional con información
graficada. Cognitivo: clasifi-
cación, comparación y asocia-
ción de dos partes de un mode-
lo. Igualación de prototipos.
Alternar con otros para utilizar
el dispositivo.
Sensorial: Tacto (motriz gruesa)
y visión.

Dispositivo

Objetivo temático

Leer y observar ilustracio

El Mural

Ilustrar las irregularidades más altas y más bajas de la Tierra.
Formación de Costa Dar a conocer los movimientos tectónicos que originaron Rica nuestro país.

La Atmósfera Recrear la estructura de la Tierra y la Atmósfera.

Las Placas y los Describir las fracciones de la Tierra y los temblores provoca-

Volcanes dos gracias al constante movimiento de sus partes.

El Reloj de la Vida Mostrar el inicio y evolución de la vida en la Tierra.

Los Volcanes de Exponer cómo se forma y de qué depende la formación de un Costa Rica volcán y los tipos de erupciones que existen.

Los Tipos de Suelo Ejemplificar cómo se clasifican los suelos para su diferenciación, según sus materiales, color, textura o zona.

Observar la recreación de los fenómenos. Cognitivo: alternar con otros para utilizar el dispositivo e interpretar de fenómenos. Sensorial: visión.
La Erosión de las
Exhibir la formación de cuevas por medio de la disolución Rocas de ciertas rocas.

Las Rocas
Fluorescentes

Mostrar cómo algunos componentes de las rocas minerales pueden emitir cierta fluorescencia cuando son expuestas a radiaciones ultravioletas.

\section{Inferir a partir de una expe- riencia cognitivo-sensorial (del cuerpo) con el objeto.}

Cognitivo: procesamiento de la información sensorial hacia una representación conceptual. Compartir el espacio con otros y coordinar las acciones para poder inferir.

Sensorial: vestibular, cenestésico y tacto.

$\begin{array}{ll}\text { El Terremoto } & \text { Exponer las normas de comportamiento adecuadas en caso } \\ \text { de sismos fuertes o terremotos. } & \text { Representar cómo ocurre un terremoto. }\end{array}$

\begin{tabular}{ll}
\hline $\begin{array}{l}\text { La Erosión de la } \\
\text { Tierra }\end{array}$ & $\begin{array}{l}\text { Experimentar los cambios de la geomorfología de los paisajes } \\
\text { por efecto de la acumulación de sedimento, causado por la } \\
\text { erosión del agua. }\end{array}$ \\
\hline El Tornado & $\begin{array}{l}\text { Sentir la fuerza del agua al ser agitada de cierta manera, para } \\
\text { la comprensión de cómo se forman los El Tornados. }\end{array}$ \\
\hline $\begin{array}{l}\text { El Arenero de los } \\
\text { Fósiles }\end{array}$ & $\begin{array}{l}\text { Excavar en la arena para la identificación de evidencias geo- } \\
\text { lógicas (fósiles) utilizadas para el análisis de especies de hace } \\
\text { miles de años, en el estudio de la evolución de la vida. }\end{array}$
\end{tabular}

Fuente: elaboración propia. 
Según se observa en la Tabla 1, los dispositivos se clasificaron por las demandas cognitivosensoriales que cada uno requiere al ser utilizado y que se definen por el diseño y posibilidades de uso de cada uno.

Debido a lo anterior, se muestra en la Tabla 2, el tiempo de atención de las personas que integran la familia: padres, madres, hijos e hijas mayores y menores según el dispositivo. En ella se puede observar el contraste de la atención focalizada, con base en los objetos que están organizados según las demandas cognitivo-sensoriales de su diseño. Para cada sujeto se indica la media (M) y la desviación estándar del tiempo (DE) invertido en ellos.

Tabla 2

Contraste de atención focalizada individual, entre miembros de la familia y dispositivos

\begin{tabular}{|c|c|c|c|c|c|c|c|c|c|}
\hline \multirow{3}{*}{$\begin{array}{l}\text { Demanda } \\
\text { cognitivo- } \\
\text { sensoriales }\end{array}$} & \multirow{3}{*}{ Dispositivo } & \multicolumn{8}{|c|}{ INTEGRANTES DE LA FAMILIA } \\
\hline & & \multicolumn{2}{|c|}{ Padres } & \multicolumn{2}{|c|}{ Madres } & \multicolumn{2}{|c|}{ Mayores } & \multicolumn{2}{|c|}{ Menores } \\
\hline & & M & $\mathrm{DE}$ & M & $\mathrm{DE}$ & M & $\mathrm{DE}$ & M & $\mathrm{DE}$ \\
\hline \multirow{4}{*}{$\begin{array}{l}\text { Leer y observar } \\
\text { ilustraciones }\end{array}$} & La Atmósfera & 13,78 & 25,48 & 2,80 & 7,99 & 1,70 & 4,44 & 3,45 & 8,27 \\
\hline & El Mural & 0,95 & 6,01 & 1,03 & 5,59 & 0,00 & 0,00 & 0,60 & 3,09 \\
\hline & $\begin{array}{c}\text { Las Placas y los } \\
\text { Volcanes }\end{array}$ & 4,08 & 16,13 & 2,20 & 9,99 & 1,88 & 8,05 & 0,90 & 5,08 \\
\hline & $\begin{array}{c}\text { La Formación de } \\
\text { C.R. }\end{array}$ & 7,03 & 20,65 & 8,18 & 27,28 & 0,63 & 2,76 & 0,05 & 0,32 \\
\hline \multirow{3}{*}{$\begin{array}{l}\text { Comparar un } \\
\text { modelo tridimen- } \\
\text { sional con infor- } \\
\text { mación graficada. }\end{array}$} & Los Tipos de Suelo. & 30,68 & 33,88 & 22,13 & 32,46 & 18,38 & 26,22 & 13,18 & 17,95 \\
\hline & El Reloj de la Vida & 11,23 & 26,58 & 17,63 & 40,09 & 6,75 & 20,34 & 7,55 & 16,29 \\
\hline & $\begin{array}{c}\text { Los } \\
\text { Volcanes de C.R. }\end{array}$ & 17,08 & 52,12 & 14,65 & 29,71 & 12,50 & 22,36 & 12,00 & 24,85 \\
\hline \multirow{2}{*}{$\begin{array}{l}\text { Observar la } \\
\text { recreación de los } \\
\text { fenómenos. }\end{array}$} & $\begin{array}{l}\text { La Erosión de las } \\
\text { Rocas. }\end{array}$ & 11,20 & 16,33 & 12,10 & 23,67 & 9,90 & 23,63 & 5,98 & 12,57 \\
\hline & $\begin{array}{l}\text { Las Rocas } \\
\text { Fluorescen-tes. }\end{array}$ & 23,73 & 49,18 & 15,80 & 18,15 & 13,18 & 24,27 & 7,30 & 13,21 \\
\hline \multirow{4}{*}{$\begin{array}{l}\text { Inferir a partir de } \\
\text { una experiencia } \\
\text { cognitivo-senso- } \\
\text { rial (del cuerpo) } \\
\text { con el objeto. }\end{array}$} & El Terremoto. & 18,45 & 21,06 & 12,60 & 14,32 & 23,60 & 18,85 & 35,38 & 27,82 \\
\hline & El Tornado. & 3,13 & 12,05 & 2,78 & 17,55 & 2,18 & 8,11 &, 25 & 1,59 \\
\hline & $\begin{array}{l}\text { El Arenero de los } \\
\text { fósiles. }\end{array}$ & 50,23 & 60,8 & 63,20 & 62,61 & 89,80 & 83,81 & 90,05 & 92,69 \\
\hline & $\begin{array}{c}\text { La Erosión de la } \\
\text { Tierra. }\end{array}$ & 67,93 & 82,99 & 92,58 & 92,44 & 127,58 & 142,07 & 120,03 & 111,71 \\
\hline
\end{tabular}

$\mathrm{M}=$ media, $\mathrm{DE}=$ desviación estándar. Fuente: elaboración propia 


\section{Resultados}

Los resultados sugieren que el tipo de actividad que se propone desde el diseño de los dispositivos, provoca en los visitantes diferencias significativas en la focalización de la atención y diferencias en la atención focalizada, considerando los dispositivos desde su diseño y las características de los integrantes de las familias.

En la Tabla 3 que se ofrece seguidamente, se observa el contraste entre el tiempo de atención focalizada de la generación adulta y joven según cada dispositivo. Los datos están organizados en orden descendente según la media de adultos.

Tabla 3

Contraste generacional, según cada dispositivo, del tiempo de atención focalizada individual

\begin{tabular}{|c|c|c|c|c|c|}
\hline \multirow{3}{*}{ Dispositivo } & \multicolumn{5}{|c|}{ Generacional } \\
\hline & \multicolumn{2}{|c|}{ Adulta } & \multicolumn{2}{|c|}{ Joven } & \multirow[b]{2}{*}{$\mathrm{P}$} \\
\hline & M & $\mathrm{DE}$ & M & $\mathrm{DE}$ & \\
\hline La Erosión de la Tierra & 160,50 & 151,692 & 247,60 & 241,91 & $\mathbf{0 , 0 0 2}$ \\
\hline El Arenero de los Fósiles & 113,43 & 109,782 & 179,85 & 170,812 & $\mathbf{0 , 0 0 1}$ \\
\hline Los Tipos de Suelo & 52,80 & 55,224 & 31,55 & 40,582 & $\mathbf{0 , 0 0 1}$ \\
\hline Las Rocas Fluorescentes & 39,53 & 60,397 & 20,47 & 32,975 & $\mathbf{0 , 0 0 2}$ \\
\hline Los Volcanes de Costa Rica & 31,73 & 67,564 & 24,50 & 45,029 & 0,366 \\
\hline El Terremoto & 31,05 & 27,226 & $\mathbf{5 8 , 9 7}$ & 39,036 & 0,000 \\
\hline El Reloj de la Vida & 28,85 & 63,959 & 14,30 & 33,098 & $\mathbf{0 , 0 3 2}$ \\
\hline La Erosión de las Rocas & 23,30 & 31,738 & 15,87 & 31,091 & 0,009 \\
\hline La Atmósfera & 16,57 & 30,244 & 5,15 & 10,899 & $\mathbf{0 , 0 1 5}$ \\
\hline La Formación de Costa Rica & 15,20 & 40,667 & 0,68 & 2,982 & $\mathbf{0 , 0 2 9}$ \\
\hline Las Placas y los Volcanes & 6,28 & 18,485 & 2,78 & 12,095 & 0,142 \\
\hline El Tornado & 5,90 & 20,867 & 2,43 & 9,083 & 0,353 \\
\hline El Mural & 1,98 & 8,084 & 0,60 & 3,087 & 0,299 \\
\hline
\end{tabular}

$\mathrm{M}=$ media, $\mathrm{DE}=$ desviación estándar, $\mathrm{P}=$ significancia. Fuente: elaboración propia.

Puede notarse que en el caso de la generación adulta (padres y madres) el dispositivo en donde pasaron en promedio más tiempo fue en la Erosión de la Tierra $(\mathrm{M}=160,50)$ y menos tiempo, en el Mural (M=1,98). El promedio de tiempo que este grupo pasó en el dispositivo de los Volcanes de Costa Rica $(\mathrm{M}=31,73)$, el Terremoto $(M=31,05)$ y el Reloj de la Vida $(\mathrm{M}=28,85)$ fue similar. En cuanto a la desviación estándar, se puede observar que la mayoría de las veces fue superior al promedio, en el caso de la Erosión de la Tierra $(\mathrm{DE}=151,692)$ y el Terremoto $(\mathrm{DE}=27,226)$ fue inferior. 
La generación joven (hijos e hijas menores y mayores) en promedio, pasó más tiempo en el Arenero $(\mathrm{M}=179,85)$ y menos tiempo en el Mural $(\mathrm{M}=0,60)$. La desviación estándar también se presentó, en la mayoría de los casos, superior al promedio de tiempo de atención, salvo en el dispositivo del Terremoto $(\mathrm{DE}=39,036)$.

Se contrastó con un t-test y se evidenció una diferencia estadísticamente significativa en el caso del tiempo de atención de la generación adulta en los siguientes dispositivos: en el Reloj de la Vida $(\mathrm{M}=28,85, \mathrm{DE}=63,959) t(39)=2,218, P=0,32$; en las Rocas Fluorescentes $(\mathrm{M}=39,53$, $\mathrm{DE}=60,397) \mathrm{t}(39)=3,276, \mathrm{P}=, 002$; en la Erosión de las Rocas $(\mathrm{M}=23,30, \mathrm{DE}=31,738) \mathrm{t}(39)=2,753$, $\mathrm{P}=, 009$; en la Formación de Costa Rica $(\mathrm{M}=15,20, \mathrm{DE}=40,667) \mathrm{t}(39)=2,271, \mathrm{P}=, 029$; en los Tipos de Suelo $(\mathrm{M}=52,80, \mathrm{DE}=55,224) \mathrm{t}(39)=3,790, \mathrm{P}=, 001$; y en la Atmósfera $(\mathrm{M}=16,57, \mathrm{DE}=30,244)$ $\mathrm{t}(39)=2,541, \mathrm{P}=, 015$.

La generación joven resultó con diferencia estadísticamente significativa en el Arenero $(\mathrm{M}=179,85, \mathrm{DE}=170,812) \mathrm{t}(39)=-3,742, \mathrm{P}=, 001$, y en el Terremoto $(\mathrm{M}=58,97, \mathrm{DE}=39,036) \mathrm{t}(39)=$ -4,662, P= ,000 y en la Erosión de la Tierra $(\mathrm{M}=247,60, \mathrm{DE}=241,91)$. Por el contrario, no se encontró diferencia significativa intergeneracional entre el tiempo de atención focalizada promedio dedicado a los demás dispositivos.

En la Tabla 4 se puede apreciar el tiempo promedio de atención focalizada en segundos según el sexo de las personas de la familia: femenino (madres e hijas menores y mayores) y masculino (padres e hijos mayores y menores) por dispositivo, y la significancia de la media.

Tabla 4

Contraste, según dispositivo, de la atención focalizada individual entre las mujeres y los hombres de las familias

\begin{tabular}{|c|c|c|c|c|c|}
\hline \multirow{3}{*}{ Dispositivo } & \multicolumn{4}{|c|}{ Según el sexo de los integrantes de la familia } & \multirow{3}{*}{$\mathrm{P}$} \\
\hline & \multicolumn{2}{|c|}{ Femenino } & \multicolumn{2}{|c|}{ Masculino } & \\
\hline & M & $\mathrm{DE}$ & M & $\mathrm{DE}$ & \\
\hline La Erosión de Tierra & 92,58 & 92,436 & 67,93 & 82,998 & ,848 \\
\hline El Arenero & 63,20 & 62,609 & 50,23 & 60,814 & ,999 \\
\hline Los Tipos de Suelo & 22,13 & 32,462 & 30,68 & 33,879 & ,966 \\
\hline El Reloj de la Vida & 17,63 & 40,096 & 11,23 & 26,572 & ,498 \\
\hline Las Rocas Fluorescentes & 15,80 & 18,149 & 23,72 & 49,178 & ,240 \\
\hline Los Volcanes de Costa Rica & 14,65 & 29,705 & 17,08 & 52,124 & ,387 \\
\hline El Terremoto & 12,60 & 14,318 & 18,45 & 21,064 & ,289 \\
\hline La Erosión de las Rocas & 12,10 & 23,665 & 11,20 & 16,326 & ,794 \\
\hline La Formación de Costa Rica & 8,18 & 27,283 & 7,03 & 20,648 & ,653 \\
\hline La Atmósfera & 2,80 & 7,997 & 13,78 & 25,479 &, 006 \\
\hline El Tornado & 2,78 & 17,551 & 3,13 & 12,049 & ,984 \\
\hline Las Placas y los Volcanes & 2,20 & 9,995 & 4,08 & 16,130 & ,406 \\
\hline El Mural & 1,03 & 5,591 & ,95 & 6,008 &, 854 \\
\hline
\end{tabular}

$\mathrm{M}=$ media, $\mathrm{DE}=$ desviación estándar, $\mathrm{P}=$ significancia. Fuente: elaboración propia. 
Con respecto al contraste según el sexo de las personas de la familia, se puede destacar que las mujeres utilizaron más tiempo en promedio en el dispositivo de la Erosión de la Tierra $(\mathrm{M}=92,58)$ y menos en el Mural (M=1,03). Se presentó una clara heterogeneidad en los promedios de duración según el dispositivo. La desviación estándar, en algunos casos, llegó a duplicar el promedio, como en el caso del Reloj de la Vida (DE=40,096) y en otros fue ligeramente inferior, como en el caso del Arenero $(\mathrm{DE}=62,609)$.

Al igual que las mujeres, los hombres atendieron, en promedio, más al dispositivo de la Erosión de la Tierra $(M=92,58)$ y menos a el Mural $(\mathrm{M=}=95)$. La desviación estándar, siguió mostrando heterogeneidad en los tiempos de atención, de esta manera en los Volcanes de Costa Rica la desviación fue superior al doble de la media $(\mathrm{DE}=52,124)$ y el más estable fue el Terremoto $(D E=21,064)$ donde la media fue sólo un poco inferior a la desviación.

Se identificó una sola diferencia estadísticamente significativa en el tiempo de atención al dispositivo de la Atmósfera para el caso de los hombres $(\mathrm{M}=13,78, \mathrm{DE}=25,479)$ t $(39)=-2,899$, $\mathrm{P}=$,006. No se encontró diferencia del tiempo promedio según el sexo en los demás dispositivos de la sala de la Tierra.

Seguidamente, en la Tabla 5, se puede observar el promedio de atención de los hijos y las hijas menores y mayores; se registra la media, la desviación estándar por dispositivo, y la significancia estadística entre menores y mayores. El cuadro está organizado según el promedio de atención de los hijos y las hijas mayores en los dispositivos de manera descendente. Obsérvese:

Tabla 5

Contraste, según dispositivo, de la atención focalizada individual entre los hijos y las hijas por edad

\begin{tabular}{|c|c|c|c|c|c|}
\hline \multirow{3}{*}{ Dispositivo } & \multicolumn{4}{|c|}{ Edad de hijos e hijas } & \multirow{3}{*}{$\mathrm{P}$} \\
\hline & \multicolumn{2}{|c|}{ Mayores } & \multicolumn{2}{|c|}{ Menores } & \\
\hline & M & $\mathrm{DE}$ & M & $\mathrm{DE}$ & \\
\hline La Erosión de la Tierra & 45,38 & 99,268 & 76,15 & 110,782 & 0,566 \\
\hline El Arenero & 34,10 & 53,132 & 59,03 & 92,679 & 0,972 \\
\hline Los Tipos de Suelo & 11,55 & 25,272 & 8,63 & 14,665 & 0,097 \\
\hline El Terremoto & 7,43 & 14,987 & 21,85 & 28,949 & 0,009 \\
\hline La Erosión de las Rocas & 4,20 & 14,046 & 4,75 & 12,066 & 0,257 \\
\hline Las Rocas Fluorescentes & 3,65 & 10,463 & 5,35 & 10,939 & 0,084 \\
\hline Los Volcanes de Costa Rica & 2,88 & 7,425 & 8,13 & 23,325 & 0,827 \\
\hline El Reloj de la Vida & 2,60 & 7,618 & 3,05 & 8,458 & 0,756 \\
\hline El Tornado & 1,23 & 6,704 &, 25 & 1,581 & 0,756 \\
\hline La Atmósfera & 0,85 & 3,752 & 1,50 & 5,579 & 0,152 \\
\hline La Formación de Costa Rica & 0,63 & 2,761 & 0,05 & 0,316 & 0,164 \\
\hline Las Placas y los Volcanes & 0,00 & 0,000 & 0,90 & 5,083 & 0,304 \\
\hline El Mural & 0,00 & 0,000 & 0,13 & 0,791 & 0,226 \\
\hline
\end{tabular}

$\mathrm{M}=$ media, $\mathrm{DE}=$ desviación estándar, $\mathrm{P}=$ significancia. Fuente: elaboración propia. 
Los hijos e hijas mayores y menores presentaron diversidad en los tiempos de atención a los dispositivos. En el caso de los hijos e hijas mayores, se notó que no visitaron del todo al Mural ni al dispositivo de las Placas y los Volcanes. El dispositivo que atendieron durante más tiempo fue el de la Erosión de la Tierra $(M=45,38)$ Este dispositivo pareciera ser también el favorito de los hijos y las hijas menores, pues fue donde pasaron más tiempo $(M=76,15)$. Sin embargo, las personas menores presentaron gran heterogeneidad en los tiempos de atención a los dispositivos.

Respecto de la desviación estándar de los hijos e hijas mayores, se observó que fue superior que la media en todos los casos. La desviación de los hijos e hijas menores presentó la misma heterogeneidad en los comportamientos.

Se confirmó la significancia estadística de la media del Terremoto para la atención individual focalizada de los hijos e hijas menores $(M=21,85, \mathrm{DE}=28,949) \mathrm{t}(39)=-2,748, \mathrm{P}=, 009$. Sin embargo, no se encontró diferencia estadística significativa en los demás dispositivos.

En la Tabla 6 que se expone a continuación, se observa la media del tiempo (en segundos) de atención focalizada según el sexo de los hijos e hijas mayores y menores por dispositivo, se indican las medias y desviaciones estándar, así como la significancia estadística de las diferencias. La tabla se organizó considerando el promedio de atención a los dispositivos de las mujeres mayores de manera descendente. 


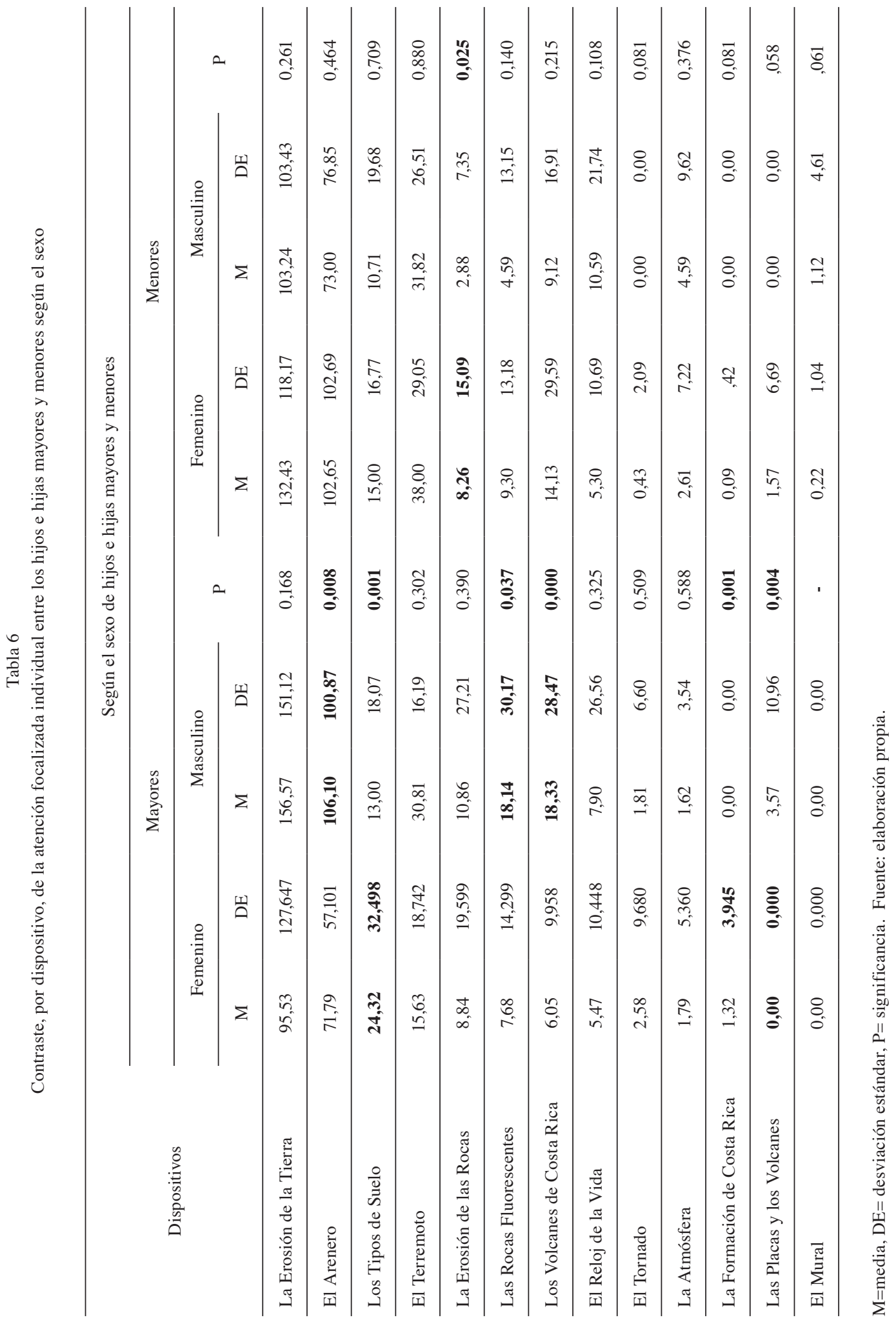


Las hijas mayores al igual que los hijos mayores, presentaron mayor promedio de atención en el dispositivo de la Erosión de la Tierra. Los hijos y las hijas mayores registraron menor desviación estándar en el Terremoto $(D E=16,185$.) Sin embargo, se observa que el resto de comportamientos fueron muy dispersos respecto de la media.

Se encontró que entre las medias de atención focalizada de los hijos e hijas mayores según el sexo, la diferencia de las medias fue estadísticamente significativa en los casos de las mujeres en Los Tipos de Suelo $(\mathrm{M}=24,32, \mathrm{DE}=32,498) \mathrm{t}(27,541)=1,342, \mathrm{P}=, 001)$; los hombres en las Rocas Fluorescentes $(\mathrm{M}=18,14, \mathrm{DE}=30,170) \mathrm{t}(29,166)=-1,422, \mathrm{P}=, 037$; los hombres en los Volcanes de Costa Rica $(\mathrm{M}=18,33, \mathrm{DE}=28,470) \mathrm{t}(25,261)=-1,855, \mathrm{P}=, 000$; las mujeres en la Formación de Costa Rica $(\mathrm{M}=1,32$, $\mathrm{DE}=3,945) \mathrm{t}(18)=1,454, \mathrm{P}=, 001$; y los hombres en las Placas y los Volcanes $(\mathrm{M}=3,57, \mathrm{DE}=10,957)$ $\mathrm{t}(20)=-1,494, \mathrm{P}=, 004$. Sin embargo, no se encontró significancia estadística según el sexo de hijos e hijas mayores en los otros dispositivos de la sala de la Tierra; tampoco entre los hijos e hijas menores. Por el contrario, el contraste mediante $t$-test registra diferencia estadística significativa según el sexo en el tiempo de atención de los demás dispositivos. Pero en relación con los promedios de atención de los hijos e hijas menores, solamente se encontró diferencia significativa en la media de las mujeres en el dispositivo la Erosión de las Rocas $(M=8,26, D E=15,091) t(38)=, 830, P=, 025$.

En la Tabla 7, se encuentra el contraste entre las medias del tiempo (en segundos) compartido e individual según dispositivo, indicando la media y la desviación estándar para cada caso y el nivel de la significancia según dispositivo. Obsérvese:

Tabla 7

Contraste de medias del tiempo compartido familiar y el tiempo individual según dispositivos

\begin{tabular}{|c|c|c|c|c|c|c|c|}
\hline \multirow{2}{*}{ Dispositivos } & \multicolumn{2}{|c|}{$\begin{array}{l}\text { Tiempo total de } \\
\text { la familia }\end{array}$} & \multicolumn{2}{|c|}{ Tiempo compartido } & \multicolumn{2}{|c|}{ Tiempo individual } & \multirow{2}{*}{$\begin{array}{c}\text { P. tiempo } \\
\text { compartido } \\
\text { e individual }\end{array}$} \\
\hline & M & DE & M & $\mathrm{DE}$ & M & $\mathrm{DE}$ & \\
\hline La Erosión de la Tierra & 408,10 & 368,302 & 145,05 & 159,105 & 263,05 & 291,824 & 0,015 \\
\hline El Arenero & 293,27 & 264,298 & 161,48 & 208,678 & 131,80 & 196,072 & 0,544 \\
\hline El Terremoto & 90,03 & 55,633 & 31,77 & 33,148 & 58,25 & 50,613 & 0,014 \\
\hline Los Tipos de Suelo & 84,35 & 90,197 & 30,45 & 48,121 & 53,90 & 70,886 & 0,074 \\
\hline Las Rocas Fluorescentes & 60,00 & 90,098 & 20,30 & 40,683 & 39,70 & 55,984 & $\mathbf{0 , 0 0 3}$ \\
\hline Los Volcanes de Costa Rica & 56,22 & 103,412 & 21,03 & 69,588 & 35,20 & 54,643 & 0,211 \\
\hline El Reloj de la Vida & 43,15 & 93,014 & 16,95 & 51,669 & 26,20 & 71,894 & 0,489 \\
\hline La Erosión de las Rocas & 39,17 & 60,473 & 12,75 & 26,312 & 26,43 & 38,591 & 0,002 \\
\hline La Atmósfera & 21,73 & 35,476 & 2,93 & 8,219 & 18,80 & 29,393 & 0,000 \\
\hline La Formación de C.R. & 15,88 & 41,095 & 2,60 & 9,440 & 7,23 & 21,658 & $\mathbf{0 , 0 1 0}$ \\
\hline Las Placas y los Volcanes & 9,05 & 27,536 & 1,83 & 10,162 & 13,28 & 32,614 & 0,090 \\
\hline El Tornado & 8,33 & 22,104 & 0,00 & 0,000 & 8,33 & 22,104 & 0,022 \\
\hline El Mural & 2,58 & 9,024 & 0,03 & 0,158 & 2,55 & 8,918 & 0,078 \\
\hline
\end{tabular}

M=media, DE= desviación estándar, P= significancia. Fuente: elaboración propia. 
Se puede observar en la tabla anterior, que la mayoría con significancia estadística se presenta para la atención focalizada individual en los casos de los dispositivos del Terremoto $(\mathrm{M}=58,25$, $\mathrm{DE}=50,613) \mathrm{t}(39)=-2,576, \mathrm{P}=, 014$; la Erosión de las Rocas $(\mathrm{M}=26,43, \mathrm{DE}=38,591) \mathrm{t}(39)=-3,254$, $\mathrm{P}=0,002$; la Atmósfera $(\mathrm{M}=18,80, \mathrm{DE}=29,393) \mathrm{t}(39)=-4,084, \mathrm{P}=0,000 ;$ la Formación de Costa Rica $(\mathrm{M}=13,28, \mathrm{DE}=32,614) \mathrm{t}(39)=-2,719, \mathrm{P}=0,010$; el Tornado $(\mathrm{M}=8,33$, $\mathrm{DE}=22,104) \mathrm{t}(39)=$ -2,382, $\mathrm{P}=0,022$; las Rocas Fluorescentes $(\mathrm{M}=39,70, \mathrm{DE}=55,984) \mathrm{t}(39)=-3,210, \mathrm{P}=0,003$; y la Erosión de la Tierra $(\mathrm{M}=263,05, \mathrm{DE}=291,824) \mathrm{t}(39)=-2,555, \mathrm{P}=0,015$. No se encontró diferencia estadísticamente significativa, entre el promedio de atención focalizada compartida e individual en otros dispositivos.

Ahora bien, considerando la clasificación de los dispositivos fundamentada en las demandas cognitivo-sensoriales de los objetos, interesa determinar la diferencia entre esa tipología y el tiempo de atención focalizada familiar. Por lo tanto, en la Tabla 8, se muestra el contraste entre la atención focalizada de cada uno de los integrantes de la familia (padres, madres, hijos e hijas mayores y menores) según tipo de dispositivo, y se indica la media y la desviación estándar. Seguidamente se ofrece la tabla:

Tabla 8

Contraste, según tipo de dispositivo, del tiempo de atención focalizada individual de cada uno de los integrantes de la familia

Integrantes de la familia

\begin{tabular}{|c|c|c|c|c|c|c|c|c|}
\hline \multirow[t]{2}{*}{ Tipo de dispositivo } & \multicolumn{2}{|c|}{ Padres } & \multicolumn{2}{|c|}{ Madres } & \multicolumn{2}{|c|}{ Mayores } & \multicolumn{2}{|c|}{ Menores } \\
\hline & M & $\mathrm{DE}$ & M & $\mathrm{DE}$ & M & $\mathrm{DE}$ & M & $\mathrm{DE}$ \\
\hline Leer y observar & 25,83 & 44,01 & 14,20 & 31,67 & 4,20 & 9,61 & 5,00 & 11,21 \\
\hline $\begin{array}{l}\text { Comparar un modelo con } \\
\text { información graficada }\end{array}$ & 58,98 & 64,21 & 54,40 & 62,57 & 37,63 & 35,22 & 32,73 & 33,07 \\
\hline $\begin{array}{l}\text { Inferir a partir de una } \\
\text { experiencia cognitivo- } \\
\text { sensorial con el objeto }\end{array}$ & 139,73 & 115,49 & 171,15 & 118,47 & 243,15 & 183,46 & 245,70 & 158,99 \\
\hline $\begin{array}{l}\text { Observar la recreación de } \\
\text { los fenómenos }\end{array}$ & 34,92 & 53,23 & 27,90 & 27,45 & 23,08 & 33,48 & 13,28 & 19,12 \\
\hline
\end{tabular}

$\mathrm{M}=$ media, $\mathrm{DE}=$ desviación estándar. Fuente: elaboración propia.

Todos los sujetos de la familia coincidieron en pasar la mayor cantidad de tiempo en los dispositivos donde se requiere inferir a partir de una experiencia cognitivo-sensorial, y tiende a ser un comportamiento homogéneo en tanto las desviaciones estándar son inferiores a la media (Padres $\mathrm{M}=139,73, \mathrm{DE}=115,49$; Madres $\mathrm{M}=171,15, \mathrm{DE}=118,47$; HY $\mathrm{M}=243,15, \mathrm{DE}=183,484$ e HN M= 246,70, $\mathrm{DE}=158,99)$. De igual manera, los miembros de la familia coinciden en pasar menos tiempo en los dispositivos de lectura y observación.

Ahora bien, al contrastar con un t-test a la generación adulta (padres y madres) con la joven (hijos e hijas menores y mayores) se encontraron diferencias las cuales se pueden observar en la Tabla 9. 
Tabla 9

Contraste, según tipo de dispositivo, del tiempo de atención focalizada individual entre la generación adulta y la Joven

\begin{tabular}{|c|c|c|c|c|c|}
\hline \multirow{3}{*}{ Tipo de dispositivo } & \multicolumn{5}{|c|}{ Generacional } \\
\hline & \multicolumn{2}{|c|}{ Adulta } & \multicolumn{2}{|c|}{ Joven } & \multirow{2}{*}{$\mathrm{P}$} \\
\hline & M & $\mathrm{DE}$ & M & $\mathrm{DE}$ & \\
\hline Leer y observar & 40,03 & 66,951 & 9,20 & 17,187 & $\mathbf{0 , 0 0 7}$ \\
\hline Comparar un modelo con información graficada & 113,38 & 112,915 & 70,35 & 64,606 & $\mathbf{0 , 0 0 0}$ \\
\hline $\begin{array}{l}\text { Inferir a partir de una experiencia cognitivo-sensorial } \\
\text { con el objeto }\end{array}$ & 310,88 & 206,678 & 488,85 & 325,787 & 0,000 \\
\hline Observar la recreación de los fenómenos & 62,83 & 68,537 & 36,35 & 45,320 & 0,000 \\
\hline
\end{tabular}

$\mathrm{M}=$ media, $\mathrm{DE}=$ desviación estándar, $\mathrm{P}=$ significancia. Fuente: elaboración propia.

Los adultos y los jóvenes enfocaron su atención principalmente en los dispositivos diseñados para inferir a partir de una experiencia cognitivo-sensorial con el objeto: los adultos en promedio pasaron 310,88 segundos en este tipo de dispositivos y fue un comportamiento bastante similar entre los sujetos de la muestra ( $\mathrm{DE}=206,68)$. Lo mismo sucedió con los y las jóvenes quienes pasaron en promedio 488,85 segundos en estos dispositivos con una $\mathrm{DE}=325,79$ que fue mucho más baja que la media, lo cual también presentó un comportamiento homogéneo. Las otras desviaciones estándar de adultos y jóvenes son superiores a la media, lo cual fue muestra de la heterogeneidad de los comportamientos ante esos tipos de dispositivos.

Se identificó una diferencia significativa de los promedios en los objetos de lectura y observación entre los adultos $(\mathrm{M}=40,03, \mathrm{DE}=66,951), \mathrm{t}(39)=3,156, \mathrm{P}=, 003$; en los de comparación de un modelo con información graficada, la significancia estadística se observa entre los adultos $(\mathrm{M}=113,38,112,915)$ $\mathrm{t}(39)=3,836, \mathrm{P}=, 000$; en los objetos de inferir a partir de una experiencia cognitivo-sensorial la significancia se localiza en los jóvenes $(\mathrm{M}=488,85, \mathrm{DE}=325,787) \mathrm{t}(39)=-4,862, \mathrm{P}=0,000$; y entre los objetos de observar la recreación de los fenómenos se encontró la diferencia estadísticamente significativa entre los adultos $(\mathrm{M}=62,83, \mathrm{DE}=68,5379), \mathrm{t}(39)=3,945, \mathrm{P}=0,000$.

En la Tabla 10, se ofrece una comparación según el sexo de los miembros familiares. Nótese la comparación:

Contraste, según tipo de dispositivo, de la atención focalizada individual entre mujeres y hombres de la familia

\begin{tabular}{|c|c|c|c|c|c|}
\hline \multirow{3}{*}{ Tipo de dispositivo } & \multicolumn{4}{|c|}{ Edad de hijos e hijas } & \multirow{3}{*}{$\mathrm{P}$} \\
\hline & \multicolumn{2}{|c|}{ Mayores } & \multicolumn{2}{|c|}{ Menores } & \\
\hline & M & $\mathrm{DE}$ & M & $\mathrm{DE}$ & \\
\hline Leer y observar & 9,61 & 4,20 & 11,21 & 5,00 & 0,672 \\
\hline Comparar un modelo con información graficada & 35,22 & 37,63 & 33,07 & 32,73 & 0,171 \\
\hline Inferir a partir de una experiencia cognitivo-sensorial con el objeto & 183,46 & 243,15 & 158,99 & 245,70 & 0,882 \\
\hline Observar la recreación de los fenómenos & 33,48 & 23,08 & 19,12 & 13,28 & 0,048 \\
\hline
\end{tabular}

$\mathrm{M}=$ media, $\mathrm{DE}=$ desviación estándar, $\mathrm{P}=$ significancia. Fuente: Elaboración propia. 
Se puede observar que existió una gran diferencia en el tiempo promedio de atención a los diferentes tipos de dispositivos, resaltando la mayoría del tiempo para los dispositivos de inferir a partir de una experiencia cognitivo-sensorial con el objeto, tanto para las mujeres $(M=416,55)$ como para los hombres $(\mathrm{M}=383,18)$. Por el contrario, los dispositivos de lectura y observación son los visitados por menos tiempo (mujeres $\mathrm{M}=18,35$ y hombres $\mathrm{M}=31,08$ ).

La desviación estándar de mujeres (DE=317,203) en este tipo de dispositivos, fue inferior a la media, lo mismo sucede en el caso de los hombres ( $\mathrm{DE}=317,195)$, de modo que en este caso, los comportamientos son homogéneos entre los sujetos de la muestra. Por el contrario, se puede observar que en los otros casos la desviación estándar fue superior a la media, lo que evidencia que hay grandes diferencias en los tiempos de atención. Ahora bien, en la tabla se indica que no existen diferencias estadísticamente significativas entre los promedios de atención según dispositivo y el sexo de las personas que integran la familia para todos los casos.

La siguiente disparidad se puede observar en la Tabla 11, donde se contrastan las medias de los tiempos de atención y se expone la desviación estándar de la atención focalizada de hijos e hijas mayores versus los menores, según su posición en la familia, y la significancia estadística. La información se organiza según tipo de dispositivo.

Contraste por tipo de dispositivo, de la atención focalizada individual entre los hijos y las hijas, según su posición en la familia

Según el sexo de los integrantes de la familia

\begin{tabular}{|c|c|c|c|c|c|}
\hline \multirow{3}{*}{ Tipo de dispositivo } & \multirow{2}{*}{\multicolumn{2}{|c|}{ Femenino }} & \multirow{2}{*}{\multicolumn{2}{|c|}{ Masculino }} & \multirow{3}{*}{$\mathrm{P}$} \\
\hline & & & & & \\
\hline & M & $\mathrm{DE}$ & M & $\mathrm{DE}$ & \\
\hline Leer y observar & 18,35 & 34,805 & 31,08 & 50,142 & 0,059 \\
\hline Comparar un modelo con información graficada & 91,23 & 96,238 & 92,50 & 97,801 & 0,932 \\
\hline $\begin{array}{l}\text { Inferir a partir de una experiencia cognitivo-sensorial con el } \\
\text { objeto }\end{array}$ & 416,55 & 317,203 & 383,18 & 317,195 & 0,599 \\
\hline Observar la recreación de los fenómenos & 45,85 & 47,136 & 53,33 & 79,337 & 0,521 \\
\hline
\end{tabular}

M=media, $\mathrm{DE}=$ desviación estándar, $\mathrm{P}=$ significancia. Fuente: elaboración propia.

Tanto los hijos y las hijas mayores $(M=183,48)$ como los hijos y las hijas menores $(M=158,99)$ pasaron mayor tiempo promedio en los dispositivos para inferir a partir de una experiencia cognitivosensorial con el objeto. Sin embargo, la desviación estándar de hijos e hijas mayores (DE=243,15) supera la media de atención, lo cual también sucede en el caso de los hijos e hijas menores (DE=245,70).

La menor cantidad de tiempo promedio de atención se destina a los dispositivos de lectura y observación, tanto mayores $(M=9,61)$ como menores $(M=11,21)$ con la fortaleza de que la desviación estándar para cada caso fue inferior a la media; lo que muestra homogeneidad en los comportamientos.

Se encontró que la diferencia según la edad fue significativa solo para los dispositivos de observación de recreación de fenómenos con los hijos y las hijas mayores $(\mathrm{M}=33,477, \mathrm{DE}=23,08) \mathrm{t}(39)=$ $2,045, \mathrm{P}=, 048$. Por el contrario, la diferencia no fue significativa para los demás dispositivos.

Otra distinción que se presenta se encuentra en la Tabla 12, ésta es la de los hijos y las hijas mayores y menores, pero según el sexo. Interesa mostrar las diferencias que se presentan según tipo de dispositivo mediante un t-test. Para ellos se indica la media, la desviación estándar y la significancia según hombres y mujeres mayores y menores. Apréciese el contraste a continuación: 


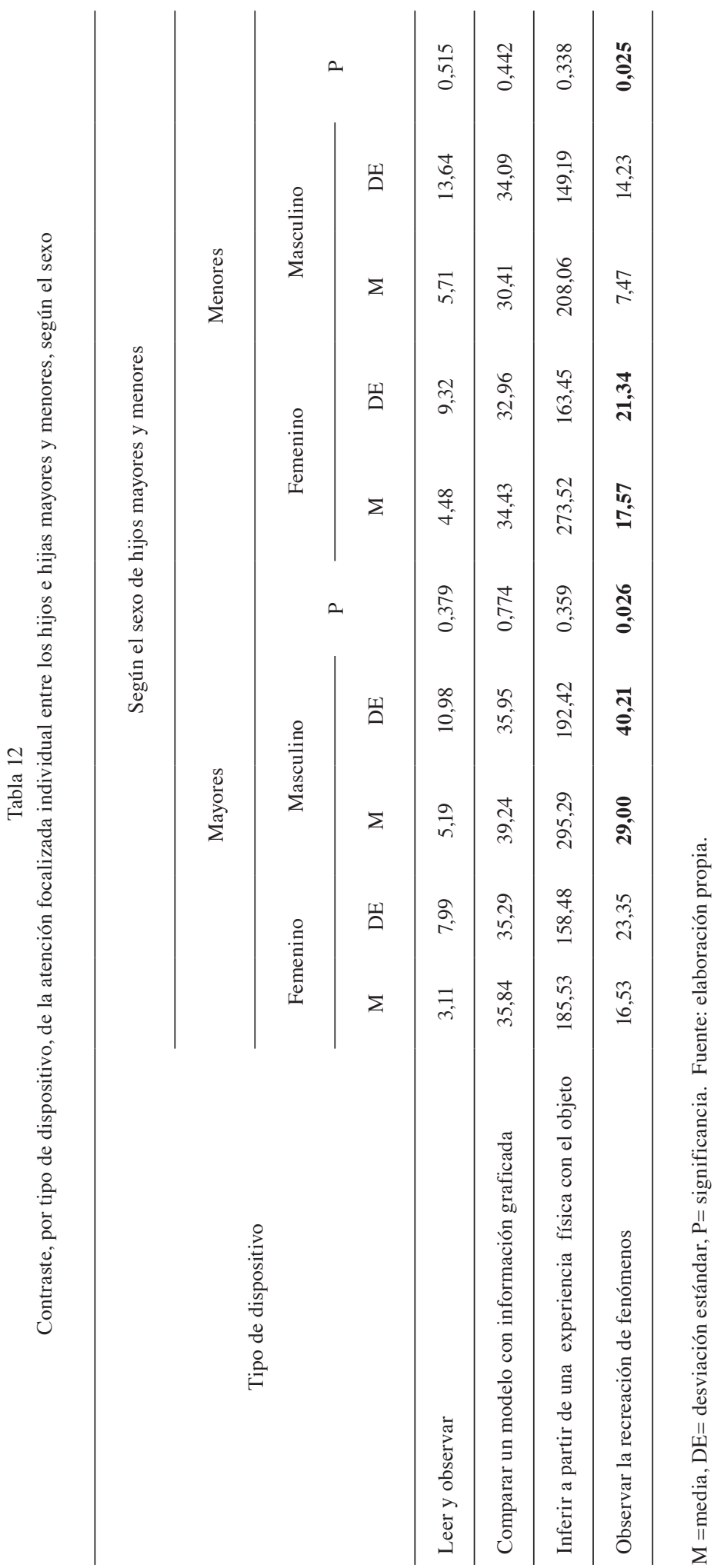


Los hijos y las hijas mayores pasaron más tiempo promedio (femenino $\mathrm{M}=185,53$ y masculino $\mathrm{M}=295$,29) en los dispositivos de inferir a partir de una experiencia cognitivo-sensorial, y menos tiempo en los dispositivos de lectura y observación (femenino $\mathrm{M}=3,11$ y masculino $\mathrm{M}=5,19$ ). Ahora bien, se notó que las mujeres pasaron más tiempo que los hombres en estos objetos.

La desviación estándar para estos dispositivos de inferencia fue inferior que la media, lo cual muestra mayor homogeneidad en los datos que en los otros casos.

Se revela que existió una diferencia significativa en la media de los hijos mayores (hombres) para los dispositivos de observación de fenómenos $(\mathrm{M}=29,00, \mathrm{DE}=40,212) \mathrm{t}(32,649)=-1,213, \mathrm{P}=0,026$. Por otro lado, no constó diferencia significativa en las demás medias de los tiempos de atención de hijas e hijos mayores. Respecto a los hijos e hijas menores, el mismo tipo de dispositivos (inferir a partir de una experiencia cognitivo-sensorial) fue el que reportó más tiempo promedio de atención para las mujeres $M=273,52$ y para hombres $M=208,06$. También en este caso, las mujeres pasaron más tiempo que los hombres en tales objetos. La desviación estándar que presentaron los datos de las mujeres en este grupo de dispositivos, fue inferior a la media $(\mathrm{DE}=163,453)$ y la desviación estándar de los hombres referido a las mismas variables, también fue inferior a la media $(\mathrm{DE}=149,193)$. El promedio de atención de las mujeres menores fue significativo para los dispositivos de observación de fenómenos $(\mathrm{M}=17,57$, $\mathrm{DE}=21,335) \mathrm{t}(37,683)=1,793, \mathrm{P}=0,025$.

No resulta diferencia estadísticamente significativa en los promedios de atención según el sexo entre los hijos y las hijas menores para los dispositivos de lectura y observación $\mathrm{t}(38)=-, 338, \mathrm{P}=0,515$; comparación de un modelo con información $\mathrm{t}(38)=, 376, \mathrm{P}=0,442$ ni en los de inferir a partir de una experiencia cognitivo-sensorial $\mathrm{t}(38)=1,299, \mathrm{P}=0,338$.

La Tabla 13 ofrece las diferencias, constatadas mediante un $t$-test, entre el tiempo promedio y la desviación estándar de atención focalizada familiar, individual y compartida y la significancia entre el tiempo familiar individual y compartido, según tipo de objeto museográfico. El tiempo individual está dado por la sumatoria del tiempo que cada uno de los integrantes de la familia pasó ante el dispositivo, mientras que el tiempo compartido es la sumatoria de los tiempos que los sujetos pasaron juntos ante el objeto.

Tabla 13

Contraste entre el tiempo compartido familiar e individual según tipo de dispositivo

\begin{tabular}{|c|c|c|c|c|c|c|c|}
\hline \multirow{2}{*}{ Tipo de dispositivo } & \multicolumn{2}{|c|}{ Tiempo total familiar } & \multicolumn{2}{|c|}{ Tiempo compartido } & \multicolumn{2}{|c|}{$\begin{array}{l}\text { Tiempo } \\
\text { individual }\end{array}$} & \multirow{2}{*}{$\begin{array}{c}\text { P. tiempo } \\
\text { compartido } \\
\text { y tiempo } \\
\text { individual }\end{array}$} \\
\hline & M & $\mathrm{DE}$ & M & $\mathrm{DE}$ & M & $\mathrm{DE}$ & \\
\hline Leer y observar & 49,23 & 75,767 & 7,38 & 16,105 & 41,85 & 63,169 & 0,000 \\
\hline $\begin{array}{l}\text { Comparar un modelo con información } \\
\text { graficada }\end{array}$ & 183,73 & 169,754 & 68,43 & 92,896 & 115,30 & 122,382 & 0,035 \\
\hline $\begin{array}{l}\text { Inferir a partir de una experiencia } \\
\text { cognitivo-sensorial con el objeto }\end{array}$ & 799,73 & 494,069 & 338,30 & 271,804 & 461,42 & 390,842 & 0,097 \\
\hline $\begin{array}{l}\text { Observar la recreación de los } \\
\text { enómenos }\end{array}$ & 99,18 & 108,173 & 33,05 & 49,393 & 66,12 & 67,616 & 0,000 \\
\hline
\end{tabular}

M=media, DE= desviación estándar, P= significancia. Fuente: elaboración propia. 
$\mathrm{Al}$ igual que en los casos anteriores, tanto en el tiempo familiar como en el tiempo compartido e individual, los dispositivos de inferir a partir de una experiencia cognitivo-sensorial con el objeto, son los que presentaron el mayor promedio de atención; en el caso de tiempo familiar en dispositivo, la media fue de 799,73 segundos; en el caso del tiempo compartido, fue de 338,30, y en el caso del tiempo individual, de 461,42.

La menor cantidad de tiempo familiar en dispositivos se ubicó en los de lectura y observación ( $M=479,23)$ y del tiempo compartido, fue también en esos dispositivos $(M=7,38)$. No obstante, sólo en este tipo de dispositivos el tiempo individual tiene un promedio superior al del tiempo compartido $(M=41,38)$

En relación con la desviación estándar en los dispositivos de inferir a partir de una experiencia cognitivo-sensorial con el objeto, tanto el tiempo familiar ( $\mathrm{DE}=494,07)$ como el tiempo compartido familiar ( $\mathrm{DE}=271,804)$ e individual $(\mathrm{DE}=390,842)$ fue inferior a la media, lo cual muestra homogeneidad en los comportamientos de atención a este tipo de dispositivos.

Se encontró que la mayoría estadísticamente significativa se presenta en la atención focalizada individual en los dispositivos de lectura y observación $(\mathrm{M}=41,85, \mathrm{DE}=63,169) \mathrm{t}(39)=-4,151, \mathrm{P}=$ ,000; comparación de un modelo con información $(\mathrm{M}=115,30, \mathrm{DE}=122,382) \mathrm{t}(39)=-2,186, \mathrm{P}=, 035$ y observación de un fenómeno $(\mathrm{M}=66,13, \mathrm{DE}=67,616) \mathrm{t}(39)=-4,341, \mathrm{P}=, 000$. Por el contrario, no se encontró diferencia entre la atención focalizada compartida e individual, en los dispositivos de inferencia $\mathrm{t}(39)=-1,703, \mathrm{P}=, 097$.

Por último, en la Tabla 14, se ofrece el contraste entre el tiempo compartido familiar y el tiempo en solitario, se indica la media y la desviación estándar por tipo de dispositivo. El tiempo en solitario consiste en el tiempo que los sujetos pasan en un dispositivo sin la compañía de otras personas de su familia.

Contraste, según tipo de dispositivo, entre el tiempo compartido familiar y el solitario

\begin{tabular}{|c|c|c|c|c|c|}
\hline \multirow{2}{*}{ Tipo de dispositivo } & \multicolumn{2}{|c|}{ Tiempo compartido } & \multicolumn{2}{|c|}{ Tiempo en solitario } & \multirow{2}{*}{$\mathrm{P}$} \\
\hline & M & DE & M & DE & \\
\hline Leer y observar & 7,38 & 16,105 & 34,48 & 52,523 & 0,001 \\
\hline Observar la recreación de los fenómenos & 33,05 & 49,393 & 33,08 & 48,184 & 0,998 \\
\hline Comparar un modelo con información graficada & 68,43 & 92,896 & 46,88 & 135,636 & 0,495 \\
\hline Inferir a partir de una experiencia cognitivo-sensorial con el objeto & 338,30 & 271,804 & 123,13 & 457,345 & 0,041 \\
\hline
\end{tabular}

M=media, $\mathrm{DE}=$ desviación estándar, $\mathrm{P}=$ significancia. Fuente: elaboración propia.

La diferencia estadísticamente significativa se localizó en los dispositivos de lectura y observación, durante el tiempo en solitario $(\mathrm{M}=34,48, \mathrm{DE}=52,523) \mathrm{t}(39)=-3,789, \mathrm{P}=, 001$; y para los dispositivos de inferir a partir de una experiencia cognitivo-sensorial, en el tiempo compartido $(\mathrm{M}=338,30$, $\mathrm{DE}=271,804) \mathrm{t}(39)=2,117, \mathrm{P}=, 04$. 


\section{Conclusiones}

Es importante tener presente que los tiempos de atención registrados son muy diferentes y heterogéneos según dispositivo y sujetos. Sin embargo, se encontró que en la visita familiar a la sala de la Tierra, unos objetos museográficos captaron la atención de las personas más que otros. Las diferencias significativas en la atención focalizada de las familias hacia los objetos museográficos, se asocian con las personas que la integran y las características de los dispositivos. Se comprueban las hipótesis planteadas en tanto existen diferencias estadísticamente significativas en la atención focalizada individual, solitaria y compartida de las familias con los objetos museográficos, pese a que la muestra es pequeña. A continuación se detalla:

Por generación, los adultos pasaron más tiempo en la Atmósfera, la Formación de Costa Rica, Los Tipos de Suelo, las Rocas Fluorescentes, el Reloj de la Vida y la Erosión de las Rocas. Las personas jóvenes utilizaron más tiempo que los adultos en los dispositivos denominados: "el Arenero" y "el Terremoto". La otra diferencia generacional encontrada fue según el tipo de dispositivos; desde esta perspectiva los adultos utilizaron más tiempo en los dispositivos de lectura y observación, comparación de modelo con información y observación de fenómenos. Por otro lado, los y las jóvenes pasaron más tiempo en los dispositivos de inferir a partir de una experiencia cognitivo-sensorial.

Según la edad (hijos e hijas mayores y menores), la diferencia de las medias de atención se encontró entre los hijos e hijas menores que pasaron más tiempo que sus hermanos y hermanas mayores en el Terremoto. Por lo demás, los comportamientos de atención son similares. En los dispositivos clasificados según demandas cognitivas y sensoriales se encontró que los hijos e hijas mayores pasaron más tiempo que los y las menores en los dispositivos de observación de fenómenos.

En cuanto a la variable de sexo de las personas de la familia, la única diferencia que señala el análisis consiste en que el dispositivo de la Atmósfera fue más utilizado por los hombres. Ahora bien, entre los hijos e hijas mayores se encontró que las mujeres pasaron más tiempo en los dispositivos de Los Tipos de Suelo y la Formación de Costa Rica. Mientras que los hombres mayores gastaron más tiempo en los Volcanes de Costa Rica y las Placas y los Volcanes. Por otro lado, los hijos y las hijas menores no registraron diferencias por sexo, en el comportamiento atencional ante los dispositivos.

No se encontró diferencia según tipo de dispositivo por demandas cognitivo-sensoriales, según el sexo entre las personas de la familia en el tiempo de duración en los dispositivos.

Con respecto a los hijos e hijas mayores, se identificó una diferencia estadística significativa para el promedio de atención de los hombres en los dispositivos de observación de fenómenos. Entre los hijos e hijas menores, el contraste mostró que las mujeres duraron más que los hombres en los dispositivos de observar fenómenos.

Otra diferencia se localizó en la atención focalizada individual y compartida ante los dispositivos, de manera que se identificaron diferencias significativas en la atención focalizada individual para los dispositivos del Terremoto, la Erosión de las Rocas, la Atmósfera, la Formación de Costa Rica, el Tornado, las Rocas Fluorescentes y la Erosión de la Tierra. En los demás dispositivos no existe diferencia entre la atención focalizada individual y la compartida. Además, se encontró que la mayoría, estadísticamente significativa, se presenta en el tiempo de la atención focalizada individual para los dispositivos de lectura y observación, comparación de un modelo con información y observación de un fenómeno.

No se encontró diferencia entre la atención focalizada compartida e individual en los dispositivos de inferir a partir de una actividad sensorial pese a que es donde se pasa más tiempo.

En relación con la atención compartida y en solitario, la diferencia estadísticamente significativa se localizó en los dispositivos de lectura y observación durante el tiempo en solitario, y en los dispositivos de inferir a partir de una experiencia cognitivo-sensorial durante el tiempo compartido. 
Existe evidencia de que los dispositivos que permiten la atención compartida son los preferidos por las familias.

Se corrobora que los dispositivos que al utilizarse demandan mayor involucramiento de sentidos captan más la atención de todos.

\section{Discusión final}

Como se pudo notar, el espectro de dispositivos que captaron la atención de los adultos, es mucho más amplio que el de los jóvenes, y pese a que los primeros consideran objetos de todos los tipos de diseños, se ubican, principalmente, en los más tradicionales, o sea, los de Lectura y Observación de imágenes o fenómenos. Esto podría estar relacionado con la experticia adquirida en el proceso de educación formal o alfabetización, lo cual acerca a los adultos más a la lectura.

En el caso de los y las jóvenes que utilizaron más tiempo que los adultos los dispositivos de Inferir a partir de una experiencia sensorial pareciera que están más interesados en experiencias y sensaciones, lo cual es mucho más afín a la novedad (Berlyne, 1972. Hwahsu y Cols., 2005. HoustonPrice y Nakai, 2004) sostenida en la actividad con los objetos de experiencias sensoriales vestibulares. Estos dispositivos requieren ser explorados utilizando varios sentidos, y por esto captan la atención de las personas jóvenes.

Esto recuerda que el fenómeno de la atención es sumamente complejo, y no sólo es un comportamiento que se pueda asociar a la edad y maduración individual, sino también es necesario comprenderla en el contexto de múltiples variables tales como estímulos preferidos y no preferidos, novedad vs. Familiaridad. De esta manera, mayor atención a un estímulo podría significar también que se está requiriendo un procesamiento de información más lento, y ante esto, la edad no necesariamente, nos da una explicación Colombo (Dic. 2002).

En relación con las diferencias de sexo indicadas, otras investigaciones han reportado cuestiones de contrastes de género en las interacciones de la niñez con las exhibiciones, entre ella Crowley y otros (May, 2001) Dierking y Falk (1994). En este caso, la exhibición estudiada es sobre ciencias naturales, y las diferencias de atención se presentan específicamente en los dispositivos de Observar la Recreación de Fenómenos Naturales, con mayor atención de parte de los hombres mayores. Esta diferencia podría ser fomentada desde el proceso de socialización de la educación formal. Crowley y otros (May, 2001) señalan que desde la escuela se promueve la diferenciación por género, suscitando entre los hombres mayor interés en el tema de ciencias naturales. Lo que resulta importante es que no se demuestra discrepancia en la atención que los dispositivos despiertan entre los niños y las niñas menores, por lo que la diferencia presentada entre las y los mayores es necesario estudiarla con mayor detenimiento. Igualmente, desde el Museo, es importante lograr captar la atención de las mujeres de esos grados de manera directa. Este es un insumo importante para futuros estudios de género y socialización, donde se requiere de aportes empíricos para determinar cuáles prácticas educativas están favoreciendo esas diferencias.

Por otro lado, aunque tanto la atención focalizada solitaria como la compartida conllevan procesos de aprendizaje, en el presente estudio queda claro que en los dispositivos donde los sujetos pasan más tiempo y donde se presentan más interacciones conjuntas son aquellos que permiten la atención compartida, que es la antesala de la actividad conjunta, y es la que permite la construcción de significados compartidos. Resulta importante entonces, considerar las características de diseño de los dispositivos de inferir a partir de una experiencia cognitivo-sensorial a la hora de planear una exhibición.

Por último, surge la interrogante de cuál sería la mejor complementación de diseños de dispositivos en una sala de exposición, cuál porcentaje debe diseñarse para uso individual y cuál para uso compartido de manera que se pueda atender ambos intereses; los de los sujetos solitarios y los de los grupos que visitan el Museo. 


\section{Recomendaciones}

Se sugieren las siguientes recomendaciones a los trabajadores de museos:

Las familias son un grupo social natural que visita el museo en una cantidad relativamente similar a la de los otros tipos de visitantes. Por lo tanto, se recomienda implementar diseños de dispositivos que faciliten la participación de los sujetos con los dispositivos y de los sujetos entre sí.

La visita en solitario al museo no resulta tan frecuente en Costa Rica, como podría serlo en Europa. Sin embargo, es importante contar con algunos recursos museográficos que atienda a este tipo de visitantes y satisfaga sus necesidades cognitivas, sin que se convierta en el tipo de diseño dominante.

Es importante tener presente que la museografía es un lenguaje comunicativo y una tecnología educativa que tiene sus particularidades, no es un libro o artículo científico diagramado en dos o tres dimensiones y nunca podría cumplir esa función. Se requiere desarrollar y pensar más la museografía como recurso didáctico y las posibilidades que tiene para potenciarla en el proceso de visita de diversos grupos y sujetos.

El guía de sala de los Museos puede ser un mediador importante del visitante solitario y los dispositivos que facilitan la atención conjunta. De la misma manera, el guía puede generar a los grupos familiares la inquietud por leer algún texto en específico dentro de la sala, mediante; enigmas, adivinanzas, juegos cortos, entre otras estrategias similares.

\section{Bibliografía}

Bakeman, Roger y Gottman, John. (1997) Observación de la interacción: introducción al análisis secuencial. Segunda edición. Cambridge Estados Unidos de Norteamérica: University Press.

Berlyne, Daniel. 1972. Estructura y función del pensamiento. Editorial Trillas. México.

Colombo, John (Dic. 2002). Infant Attention Grows Up: The Emergence of a Developmental Cognitive Neuroscience Perspective. [El crecimiento de la atención infantil: la emergencia del desarrollo cognitivo desde una perspectiva en Neurociencias.] Current Directions in Psychological Science. Blackwell Publishing, Inc. 11 (6), 196-200.

Colomina, Rosa (1996). Interacció social i influència educativa en el context familiar. [Interacción social e influencia educativa en el contexto familiar]. Tesis doctoral presentada en el Departamento de Psicología Evolutiva y de la Educación de la Universidad de Barcelona. España.

Crowley, Kevin, Callanam, Maureen, Tenembaum, Harriet y Allen, Elizabeth. (May, 2001) Parents Explain More Often to Boys Than to Girls During Shared Scientific Thinking. [Los padres explican más frecuentemente a los niños que a las niñas, mientras comparten pensamientos científicos.] Psicological Science. 12(3), 258-611.

Dierking, Lynn y Falk, John (1994). Family Behavior and Learning in Informal Science Settings: A Review of the Research. [Comportamiento familiar y aprendizaje en contextos informales de ciencias: una revision de la investigación.] Science Education, 78(1), 57-72.

Houston-Price, Carmel y Nakai, Satsuki (Septiembre, 2004) Distinguishing Novelty and Familiarity Effects in Infant Preference Procedures. [Distinguiendo los efectos de la novedad y la familiaridad en las preferencias de los procedimientos.] Infant and Child Development, 13, 341-348.

Hwahsu, SH.; Lee, FL. y Wu, MC. 2005. Designing Action Games for Appealing to Buyers. [Diseñando juegos de acción para atraer a compradores.] Cyberpsychology \& Behavior,_, 8 (6), 585 - 591.

Mas, María Teresa. (2003). L'atenció Conjunta dels 10 Als 28 Mesos D'edat de L'infant. [La atención conjunta de los 10 a los 28 meses de edad del niño.] Tesis para optar por el grado de Doctora. Dpto. de Psicología de la Educación, Universidad de Barcelona. España.

Quera, Vincent. (1997) Los métodos observacionales en la Etología. En: Fernando Peláez y Joaquim Veà (compiladores). Etología bases biológicas de la conducta animal y humana. España: Ediciones Pirámide. 
Rodríguez, Keilyn. (2008) La participación guiada de padres y madres con sus hijas o hijos de I y II ciclo escolar en la visita familiar al Museo de los niños de Costa Rica. Tesis sometida a la consideración de la comisión del Programa de Doctorado en Educación para Optar por el grado de Doctora en Educación. Facultad de Educación. Universidad de Costa Rica. Costa Rica.

Vilnitzki, Laura (2005). Reporte de avance de tesis doctoral. Istituto di Comunicazione e formazione (ICEF). Facoltá Scienze della Comunicazione. Università della Svizzera italiana (USI). Via Buffi 13 - 6904 Lugano-Svizzera. 
